\section{$2.5 \mathrm{~V} 35 \mathrm{dBm}$ IIP3 $75 \mathrm{MHz}$ sixth-order active RC filter}

E. De Backer, J. Bauwelinck, C. Mélange, E. Matei, P. Ossieur, X.-Z. Qiu, J. Vandewege and S. Horvath

A differential sixth-order Butterworth Sallen-Key lowpass filter in $0.25 \mu \mathrm{m}$ SiGe BiCMOS, using a $2.5 \mathrm{~V}$ supply, is presented. The filter has a $75 \mathrm{MHz}$ cutoff frequency and an attenuation of more than $20 \mathrm{~dB}$ at a stopband frequency of $148 \mathrm{MHz}$. The third-order intercept point (IIP3) is $35 \mathrm{dBm}$, providing excellent linearity.

Introduction: A differential sixth-order Butterworth Sallen-Key lowpass filter is used as a reconstruction filter for new generation power line transmitters [1]. $0.25 \mu \mathrm{m}$ SiGe BiCMOS was chosen for a high-performance broadband powerline front-end IC design. To avoid the disturbance of other available services, deep notches are needed in a multicarrier scheme, leading to a high linearity requirement. An active RC filter uses operational amplifiers (opamp) in combination with resistors and capacitors to provide a LRC-like behaviour.

Filter design: The Sallen-Key filter is a good choice when gain accuracy is important. A unity gain stage is used with a low quality factor $Q$. A high $Q$ response would suffer from additional noise caused by peaking. Advantages of the unity gain operation are the inherent stability of the feedback loops around the unity gain buffer and the low $Q$ sensitivity to resistor and capacitor variations.

A Butterworth response was chosen owing to its small group delay variation and monotonically increasing attenuation with frequency in the stopband. The aim of the filter is to remove unwanted frequencies after DAC. With an input signal bandwidth extending to $60 \mathrm{MHz}$ and a DAC sample rate of $208 \mathrm{Msps}$, the first aliasing image would be at $148 \mathrm{MHz}$ and must be attenuated by more than $20 \mathrm{~dB}$ as required for the application. A disadvantage of a RC filter is that the capacitive and resistive elements suffer from process variations, resulting in different cutoff frequencies. The attenuation at the stopband frequency $f_{s}(148 \mathrm{MHz})$ is specified as $20 \mathrm{~dB}$, so the minimum order $N$ for a Butterworth approximation fulfils $-20 N \log \left(f_{s} / f_{B W}\right)>20 \mathrm{~dB}$. According to this equation (with $f_{B W} 60 \mathrm{MHz}$ ), a filter order of three would be sufficient. However, simulations showed that capacitor and resistor variations with temperature and process corners require a sixth-order filter (with a cutoff frequency of $75 \mathrm{MHz}$ ) to meet the specification. Fig. $1 a$ shows the proposed sixth-order Sallen-Key lowpass filter. It consists of a cascade of three second-order sections. Lower $Q$ stages are placed ahead of higher $Q$ stages to prevent opamp output saturation due to high gain peaking and to attenuate high-frequency out-of-band signals and input noise. High linearity is obtained, up to high frequencies, by feedback linearisation of the unity gain closed-loop buffers [2].

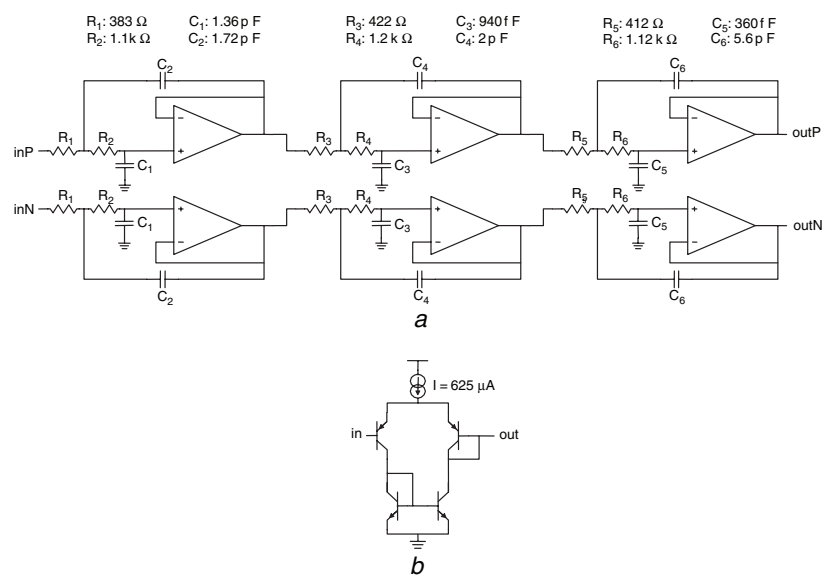

Fig. 1 Sixth-order differential Sallen-Key filter

a Schematic diagram

$b$ Unity gain buffer

The conventional approach for a Sallen-Key filter has a singleended output. However, a fully differential implementation was chosen, because a differential design can halve the signal swing and lower the IM3 (third-order intermodulation) distortion by $12 \mathrm{~dB}$. A straightforward way to implement a differential filter is to use two matched single-ended active filters. Fig. $1 b$ shows the unity gain buffer. The unity gain buffer, with an open loop gain bandwidth product of $11.64 \mathrm{GHz}$, is a collector-coupled differential pair with an inverted input terminal and output terminal short circuit [3]. Thanks to the use of a closed-loop gain stage, a low gain error is achieved. The mismatch between both paths is low enough to be able to take advantage of differential signalling. The DC-offset caused by mismatch would be no problem for the application because AC coupling is used and because the desired frequencies are above $1.6 \mathrm{MHz}$ [1]. An additional advantage of this singleended opamp is that it needs no common-mode feedback circuit. The optimum output common-mode voltage is $900 \mathrm{mV}$ and it provides the best linearity (highest open-loop gain). Choosing the $\mathrm{R}$ and $\mathrm{C}$ values requires a trade-off between noise, linearity and power consumption. The resistors in the filter will generate thermal noise, so one would like to minimise the resistance where noise voltage is important (all resistors are smaller than $1.2 \mathrm{k} \Omega$ ). However, for the same cutoff frequency, if the resistance is decreased the capacitance has to increase. There are a number of disadvantages in increasing the capacitances. First, the area will increase. Second, the capacitors $\mathrm{C}_{2}, \mathrm{C}_{4}$ and $\mathrm{C}_{6}$ will load down the amplifiers. This decreases the slew rate of the amplifier, and the increased current to drive the capacitor will increase the distortion and the power dissipation. Consequently, the selection of the $\mathrm{R}$ and $\mathrm{C}$ values was an iterative process using Cadence to simulate the circuit performance and FilterPro [4] to calculate different sets of $\mathrm{R}$ and $\mathrm{C}$ values. The implemented values can be found in Fig. 1a. The noise of the filter is very low and mostly determined by the resistors.

Experimental results: Fig. 2 shows the measured AC characteristic of the filter. The $3 \mathrm{~dB}$ bandwidth of the filter is $75 \mathrm{MHz}$ and the attenuation for the stopband frequency of $148 \mathrm{MHz}$ is $26 \mathrm{~dB}$. The attenuation in the passband $(0.7 \mathrm{~dB})$ is due to losses in the test setup (de-embedding the filter gives a passband attenuation of $0.16 \mathrm{~dB}$ ). In Fig. 3 the IM3 is shown for different input signals in function of the frequency. For example, for two differential input tones of $-2 \mathrm{dBm}$ ( $4 \mathrm{dBm}$ input power) spaced at $10 \mathrm{kHz}$ around $60 \mathrm{MHz}$, the IM3 distortion is about $-58.17 \mathrm{dBc}$. Fig. 4 shows the measured two-tone third-order input referred intercept point (IIP3), which is $35 \mathrm{dBm}$, providing very high linearity. The input referred noise is $-144.63 \mathrm{dBm} / \mathrm{Hz}$ or $13.122 \mathrm{nV} / \operatorname{sqrt}(\mathrm{Hz})$ at $10 \mathrm{MHz}$. This is well below the typical power line noise floor of $-130 \mathrm{dBm} / \mathrm{Hz}$. The circuit uses a die area of $0.169 \mathrm{~mm}^{2}$. The power consumption of the filter is $15 \mathrm{~mW}$ at $2.5 \mathrm{~V}$ supply. Table 1 summarises the measured performance of the filter, which is compared with [5-7]. The presented filter provides a higher bandwidth with a higher filter order, a significantly, higher IIP3, a smaller area and low noise compared to [5-7].

The power consumption is higher than [5-7]; however, this is a less stringent requirement for a power line front-end (and the bandwidth is 3-14 times higher). By using some extra power, the linearity has been increased significantly, this is the main issue for multicarrier power line communications.

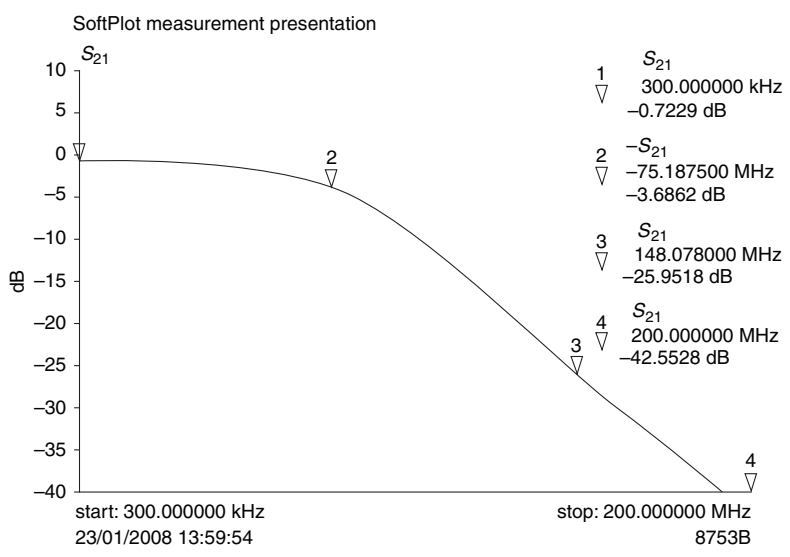

Fig. 2 AC characteristic 


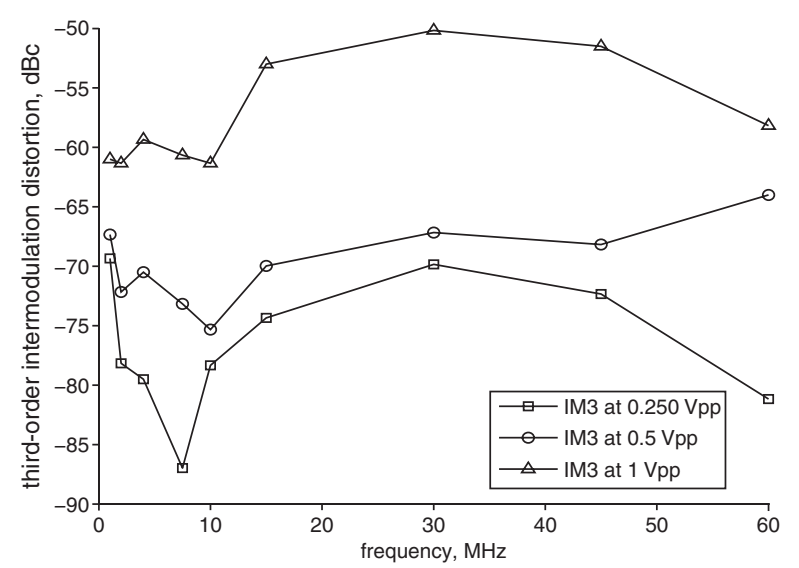

Fig. 3 Third-order intermodulation distortion

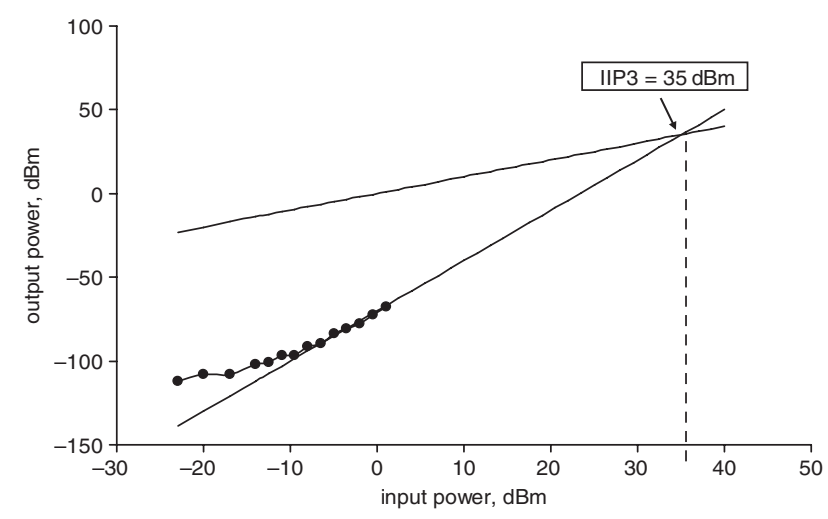

Fig. 4 Two-tone test at $60 \mathrm{MHz}$

Table 1: Comparison of filter implementation

\begin{tabular}{|c|c|c|c|c|}
\hline Reference & {$[5]$} & {$[6]$} & {$[7]$} & This work \\
\hline Technology & $\begin{array}{c}0.12 \mu \mathrm{m} \\
\text { CMOS }\end{array}$ & $\begin{array}{c}0.18 \mu \mathrm{m} \\
\text { CMOS }\end{array}$ & $\begin{array}{c}0.13 \mu \mathrm{m} \\
\text { CMOS }\end{array}$ & $\begin{array}{c}0.25 \mu \mathrm{m} \\
\text { SiGe BiCMOS }\end{array}$ \\
\hline Filter order & 5 & 4 & 5 & 6 \\
\hline Area $\left(\mathrm{mm}^{2}\right)$ & 0.25 & 0.52 & 0.20 & 0.169 \\
\hline Supply (V) & 1 & 1.8 & 1.5 & 2.5 \\
\hline $\begin{array}{c}\text { Power consumption } \\
(\mathrm{mW})\end{array}$ & 6.1 & 4.1 & 11.3 & 15 \\
\hline DC-gain (dB) & 0 & -3.5 & 2 & 0 \\
\hline BW (MHz) & 5 & 10 & $19.7 / 8.9$ & 75 \\
\hline IIP3 (dBm) & 20 & 17.5 & 18.3 & 35 \\
\hline Noise (nV/sqrt(Hz)) & $\mathrm{x}$ & 7.5 & 30 & 13.2 \\
\hline
\end{tabular}

Conclusions: A $70 \mathrm{MHz}$ sixth-order Butterworth active RC filter is presented. A very high linearity (IIP3 $35 \mathrm{dBm}$, IM3 below $-50 \mathrm{dBc}$ for $1 \mathrm{Vpp}$ up to $60 \mathrm{MHz})$ and low noise $(13 \mathrm{nV} / \operatorname{sqrt}(\mathrm{Hz}))$ has been achieved. Compared to previous publications, the presented filter consumes a lower area and achieves a much higher linearity, notwithstanding a higher bandwidth and higher filter order.

Acknowledgments: This work was supported by the European Commission in the framework of FP6 (IST project POWERNET). The authors thank the COT Business Unit of Wire line Infrastructure Division of STMicroelectronics for providing technical support, and STMicroelectronics for the chip fabrication.

(C) The Institution of Engineering and Technology 2008 28 January 2008

Electronics Letters online no: 20080254 doi: 10.1049/el:20080254

E. De Backer, J. Bauwelinck, C. Mélange, E. Matei, X.-Z. Qiu and J. Vandewege (INTEC/IMEC, Ghent University, St. Pietersnieuwstraat 41, 9000 Gent, Belgium)

E-mail: els.debacker@intec.ugent.be

P. Ossieur (Post doctoral fellow FWO-Vlaanderen, Belgium)

S. Horvath (ACN Advanced Communications Networks SA, rue du Puits-Godet 8a, 2000, Neuchâtel, Switzerland)

\section{References}

1 Bauwelinck, J., De Backer, E., Mélange, C., Matei, E., Ossieur, P., Qiu, X.-Z., Vandewege, J., and Horvath, S.: 'High dynamic range $60 \mathrm{MHz}$ powerline front-end IC', Electron. Lett., 2008, 44, (5), pp. 348-349

2 Riley, T., and Dell'Aera, S.: 'Sampling IF filters and the return of the superheterodyne receiver', Microw. J., 2005, 48, pp. 86-100

3 Palmisano, G., Palumbi, G., and Pennisi, S.: 'High-performance and simple CMOS unity-gain amplifier', IEEE Trans. Circuits Syst. I, Fundam. Theory Appl., 2000, 47, (3), pp. 406-410

4 Bishop, J., Trump, B., and Stitt, M.: 'FilterPro MFB and Sallen-Key lowpass filter design program', Application Report, 2001

5 Vasilopoulos, A., Vitzilaios, G., Theodoratos, G., and Papananos, Y.: 'A low-power wideband reconfigurable integrated active-RC filter with $73 \mathrm{~dB}$ SFDR', IEEE J. Solid-State Circuits, 2006, 41, (9), pp. $1997-2008$

6 D'Amico, S., Conta, M., and Baschirotto, A.: 'A $4.1 \mathrm{~mW} 10 \mathrm{MHz}$ fourth-order source-follower based continuous-time filter with 79-dB DR', IEEE J. Solid-State Circuits, 2006, 41, (12), pp. 2713-2719

7 Kousai, S., Hamada, M., Ito, R., and Itakura, T.: 'A 19.7 MHz, Fifthorder active-RC Chebyshev LPF for Draft IEEE802.11n with automatic quality-factor tuning scheme', IEEE J. Solid-State Circuits, 2007, 42, (11), pp. 2326-2337 